\title{
A convenient multiplex PCR system for the detection of dystrophin gene deletions: a comparative analysis with cDNA hybridisation shows mistypings by both methods
}

\author{
Stephen Abbs, Shu C Yau, Suzanne Clark, Christopher G Mathew, Martin Bobrow
}

\begin{abstract}
Existing reactions for the multiplex PCR amplification of exons in the dystrophin gene have been modified to produce two multiplex reactions which separately cover the $5^{\prime}$ and $3^{\prime}$ major deletion 'hotspots' in the gene, and together detect approximately $\mathbf{9 8 \%}$ of all deletions detectable by Southern cDNA hybridisation.

A comparative study of 148 patients showed mistypings in both the cDNA hybridisation data (4\%) and the PCR analysis (1.2\%). We suggest means of circumventing the underlying problems in order to avoid mistyping and subsequent misdiagnosis, and conclude that, with appropriate precautions, multiplex PCR amplification can be the method of choice for detecting deletions in the dystrophin gene.
\end{abstract}

Patients with Duchenne or Becker muscular dystrophy (DMD/BMD) exhibit pathological deletions within the dystrophin gene in approximately $60 \%$ of cases ${ }^{1-3}$ and a duplication can be detected in an additional $6 \%{ }^{4}$ Detection of the mutation in an affected subject can greatly enhance the accuracy of prediction of carrier status for female relatives and of subsequent prenatal diagnostic testing. Where no deletion or duplication can be identified, the nature and location of the causative mutations are unknown at present and carrier risks and prenatal diagnoses are performed by analysis of restriction fragment length polymorphisms. ${ }^{56}$ Linkage analysis, however, carries a significant error rate, resulting from the $12 \%$ recombination frequency observed within the dystrophin

Division of Medical and Molecular Genetics, Paediatric Research Unit, United Medical and Dental Schools, Guy's Hospital, London SE1 9RT.

S Abbs, S C Yau, S Clark, C G Mathew, M Bobrow Correspondence to $\mathrm{Mr}$ Abbs.

Received for publication 28 November 1990.

Accepted for publication 21 December 1990. gene. ${ }^{7}$ The identification of the mutation is therefore the primary task in the analysis of Duchenne or Becker muscular dystrophy families requesting genetic counselling.

$A$ rapid and efficient method of screening for deletions was introduced by Chamberlain $e t a l{ }^{8}$ by using the polymerase chain reaction (PCR) ${ }^{9}$ to analyse six frequently deleted exons simultaneously in a multiplex amplification reaction. Three additional exons were subsequently incorporated into the reaction, ${ }^{10}$ enabling the detection of approximately $80 \%$ of deletions that were previously detectable with cDNA probes. The reliability of this multiplex has been verified in a multicentre collaborative trial (Chamberlain $e t a l$, in preparation). A multiplex of a further nine exons developed by Beggs et al ${ }^{11}$ complements this reaction to increase the detection frequency to $94.5 \%$ (table 1 ).

We have modified the amplifications included in these multiplexes and reorganised them into two reactions which separately cover the two major deletion prone regions in the dystrophin gene. Additionally, the frequency of deletions detectable has been increased to $98 \%$.

We report a study of 98 patients with known deletions and of 50 non-deleted patients, analysed by both Southern cDNA hybridisation and by multiplex PCR amplification. Discrepancies between the results obtained by the two different methods have been fully investigated and we suggest means of circumventing

Table 1 Deletion detection frequencies using multiplex PCR amplification. Figures are derived from the analysis of our own data from 222 deletions, plus the 273 deletions reported by Koenig et al. ${ }^{12}$

\begin{tabular}{|c|c|c|}
\hline Multiplex reaction & $\begin{array}{l}\text { Number of deletions } \\
\text { detectable by PCR }\end{array}$ & $\%$ detection \\
\hline $\begin{array}{lll}\text { Chamberlain et al } & l^{8} & 10 \\
\text { Chamberlain et } a l^{8} & 10\end{array}$ & $388 / 495$ & $78 \cdot 3$ \\
\hline $\begin{array}{l}\text { + Beggs et } \text { al }^{11} \\
5^{\prime} \\
3^{\prime} \\
5^{\prime}+3^{\prime}\end{array}$ & $\begin{array}{l}468 / 495 \\
111 / 495 \\
379 / 495 \\
484 / 495\end{array}$ & $\begin{array}{l}94 \cdot 5 \\
22 \\
76 \cdot 5 \\
97 \cdot 7\end{array}$ \\
\hline
\end{tabular}


HindIII fragments

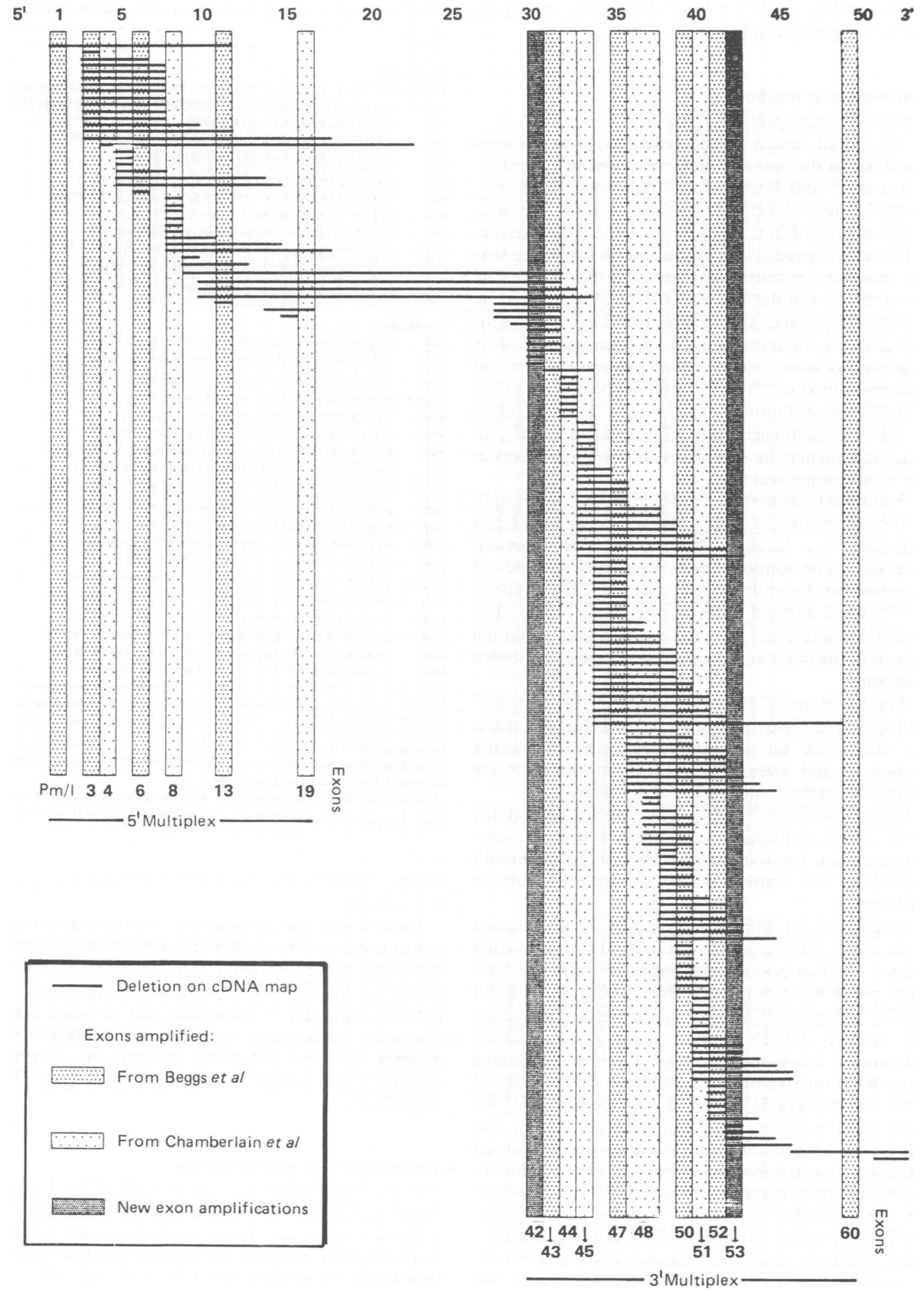

Figure 1 Detection of deletions by multiplex PCR amplification. Horizontal lines represent separate deletions, ${ }^{3}$ mapped by Southern hybridisation of cDNA probes to HindIII digests. Vertical bars represent the locations of exons amplified in multiplex reactions. 
the underlying problems in order to avoid mistyping and subsequent misdiagnosis.

\section{Materials and methods \\ MULTIPLEX PCR AMPLIFICATION}

In the initial design of the reactions, the 18 exons amplified in the two multiplex reactions of Chamberlain $e t a l^{810}$ and Beggs $e t a l^{11}$ were divided into two groups of nine, depending on their $5^{\prime}$ or more central (3') location within the dystrophin gene. Examination of the sizes of products of amplification suggested that the products within each group could readily be resolved by standard horizontal electrophoresis by altering the product size of exon 44 . The $5^{\prime}$ primer for this exon was therefore moved 158 base pairs $3^{\prime}$ of its original location, using the sequence data of Chamberlain et al. ${ }^{8}$ The location of the $5^{\prime}$ primer for exon 45 was also moved to reduce the size of product by $240 \mathrm{bp}$ (sequence from Chamberlain et $a l^{8}$ ), in order to enhance the overall separation of products in the $3^{\prime}$ multiplex reaction.

Additional primers were designed for the amplification of exons 42 and 53 using the published cDNA sequence $^{13}$ to increase the number of deletions detected. The amplifications of exons 12 and 17 (included in the multiplex reaction of Chamberlain $e t$ $a l^{810}$ ) were omitted from our reactions, since they would not detect any reported deletion that would not extend to the other exons amplified in the 5 ' multiplex reaction.

The locations of exons amplified in the $5^{\prime}$ and $3^{\prime}$ multiplex reactions, with respect to cDNA deletions, are shown in fig 1 . The oligonucleotide primer sequences and sizes of amplification products are detailed in table 2 and fig 2, respectively.

Primers were synthesised on an Applied Biosystems 391A DNA synthesiser, deprotected, ethanol precipitated, and resuspended in Tris-EDTA $(10 \mathrm{mmol} / \mathrm{l}$ Tris-HCl, $0.1 \mathrm{mmol} / \mathrm{l}$ EDTA) without further purification.

Amplifications were performed in $50 \mu \mathrm{l}$ volumes containing: $250 \mathrm{ng}$ genomic DNA; $0.5 \mu \mathrm{mol} / \mathrm{l}$ each primer; $1 \times$ Taq polymerase buffer $(67 \mathrm{mmol} / 1$ Tris$\mathrm{HCl}, \mathrm{pH} 8.8,16.6 \mathrm{mmol} / \mathrm{l}\left(\mathrm{NH}_{4}\right)_{2} \mathrm{SO}_{4}, 6.7 \mathrm{mmol} / \mathrm{l}$ $\mathrm{MgCl}_{2}, 170 \mu \mathrm{g} / \mathrm{ml} \mathrm{BSA}, 10 \mathrm{mmol} / 12$-mercaptoethanol); $0.5 \mathrm{mmol} / 1 \mathrm{dNTPs}$ and 5 units of Taq DNA polymerase (Amplitaq, Cetus). Cycling conditions were: $94^{\circ} \mathrm{C}$ for five minutes, followed by 23 cycles of $94^{\circ} \mathrm{C}$ for 48 seconds, $60^{\circ} \mathrm{C}$ for 48 seconds, $72^{\circ} \mathrm{C}$ for three minutes, with a final extension of $72^{\circ} \mathrm{C}$ for five minutes. The products of amplification were visualised directly by electrophoresis of 10 to $12 \mu \mathrm{l}$ of product in horizontal polyacrylamide minigels $(5 \%$ acrylamide for the $3^{\prime}$ multiplex, or $7 \%$ for the $5^{\prime}$ multiplex, $1 \times$ TBE $[89 \mathrm{mmol} / 1$ Tris- $\mathrm{HCl}, 89 \mathrm{mmol} / 1$ boric acid, 2 mmol/1 EDTA, $\mathrm{pH} 8$ ], $0.5 \mu \mathrm{g} / \mathrm{ml}$ ethidium bromide, in $1 \times$ TBE running buffer, at $90 \mathrm{~mA}$ for one to two
Table 2 Sequences of primers used in the multiplex reactions.

\begin{tabular}{ll}
\hline \multicolumn{1}{c}{ Sequence (5'-3') } \\
\hline Primer & \\
\hline 5' reaction & \\
PmF & GAAGATCTAGACAGTGGATACATAACAAATGCATG \\
PmR & TTCTCCGAAGGTAATTGCCTCCCAGATCTGAGTCC \\
3F & TCATCCATCATCTTCGGCAGATTAA \\
3R & CAGGCGGTAGAGTATGCCAATGGAAATCA \\
4F & TTGTCGGTCTCCTGCTGGTCAGTG \\
4R & CAAAGCCCTCACTCAAACATGAAGC \\
6F & CCACATGTAGGTCAAAAATGTAATGAA \\
6R & GTCTCAGTAATCTTCTTACCTATGACTATGG \\
8F & GTCCTTTACACACTTTACCTGTTGAG \\
8R & GGCCTCATTCTCATGTTCTAATTAG \\
13F & AATAGGAGTACCTGAGATGTAGCAGAAAT \\
13R & CTGACCTTAAGTTGTCTTCCAAAGCAG \\
19F & TTCTACCACATCCCATTTCTTCCA \\
19R & GATGGCAAAAGTGTTGAGAAAAAGTC \\
3' reaction & \\
42F & CACACTGTCCGTGAAGAAACGATGATG \\
42R & TTAGCACAGAGGTCAGGAGCATTGAG \\
43F & GAACATGTCAAAGTCACTGGACTTCATGG \\
43R & ATATATGTGTTACCTACCCTTGTCGGTCC \\
44F & GTTGTGTGTACATCGTAGGTGTGTA \\
44R & TCCATCACCCTTCAGAACCTGATCT \\
45F & CTTTCTTTGCCAGTACAACTGCATGTG \\
45R & CATTCCTATTAGATCTGTCGCCCTAC \\
47F & CGTTGTTGCATTTGTCTGTTTCAGTTAC \\
47R & GTCTAACCTTTATCCACTGGAGATTTG \\
48F & TTGAATACATTGGTTAAATCCCAACATG \\
48R & CCTGATAAAGTCTTCCTTACCACAC \\
50F & CACCAAATGGATTAAGATGTTCATGAAT \\
50R & TCTCTCTCACCCAGTCATCACTTCATAG \\
51F & GAAATTGGCTCTTTAGCTTGTGTTC \\
51R & GGAGAGTAAAGTGATTGGTGGAAAATC \\
52F & AATGCAGGATTGGAACAGAGGCGTCC \\
52R & TTCGATCCGTAATGATTGTCTAGCCTC \\
53F & TTGAAAGATTCAGATCAGTGGGATG \\
53R & CTTGGTTTCTGTGATTTTCTTTTGGATTG \\
60F & AGGAGAAATTGCGCCTCTGAAAGAGAACG \\
60R & CTGCAGAAGCTTCCATCTGGTGTTCAGG \\
\hline &
\end{tabular}

Primers are named by the exons they amplify: $F$ and $R=$ forward and reverse in relation to the cDNA sequence; Pm amplifies the muscle specific promotor plus a quarter of exon 1 . Primer sequences are as in references of Chamberlain et al ${ }^{10}$ and Beggs et al, ${ }^{11}$ except for: (a) additional primers-sequence from $\mathrm{cDNA}^{13}$; (b) primers modifiedsequence from Chamberlain et al. ${ }^{8}$

${ }^{*}$ Primer $44 \mathrm{~F}$ should be replaced by $44 \mathrm{~F} 2$ since the sequence of $44 \mathrm{~F}$ is incorrect (see text). Sequence of 44F2, with correction underlined: GTTGTGTGTACATGCTAGGTGTGTA.

hours). Additional cycles were performed on samples as necessary.

Each of the two reactions was verified for its use as a diagnostic test by performing a blind analysis on 98 randomly chosen patients with deletions within the region covered by the multiplex being tested (48 patients with the $5^{\prime}$ multiplex and 50 with the $3^{\prime}$ reaction). Additionally, a group of $50 \mathrm{DMD} / \mathrm{BMD}$ patients with no detectable deletion by Southern analysis were analysed with both multiplex PCR amplifications.

ADDITIONAL PCR AMPLIFICATIONS

For the verification of results that showed a discrepancy between the Southern cDNA data and PCR multiplex data, additional oligonucleotide primers were designed using the published $\mathrm{cDNA}$ sequence ${ }^{13}$ specifically to amplify exons $3,7,9$, and 44 . Each 


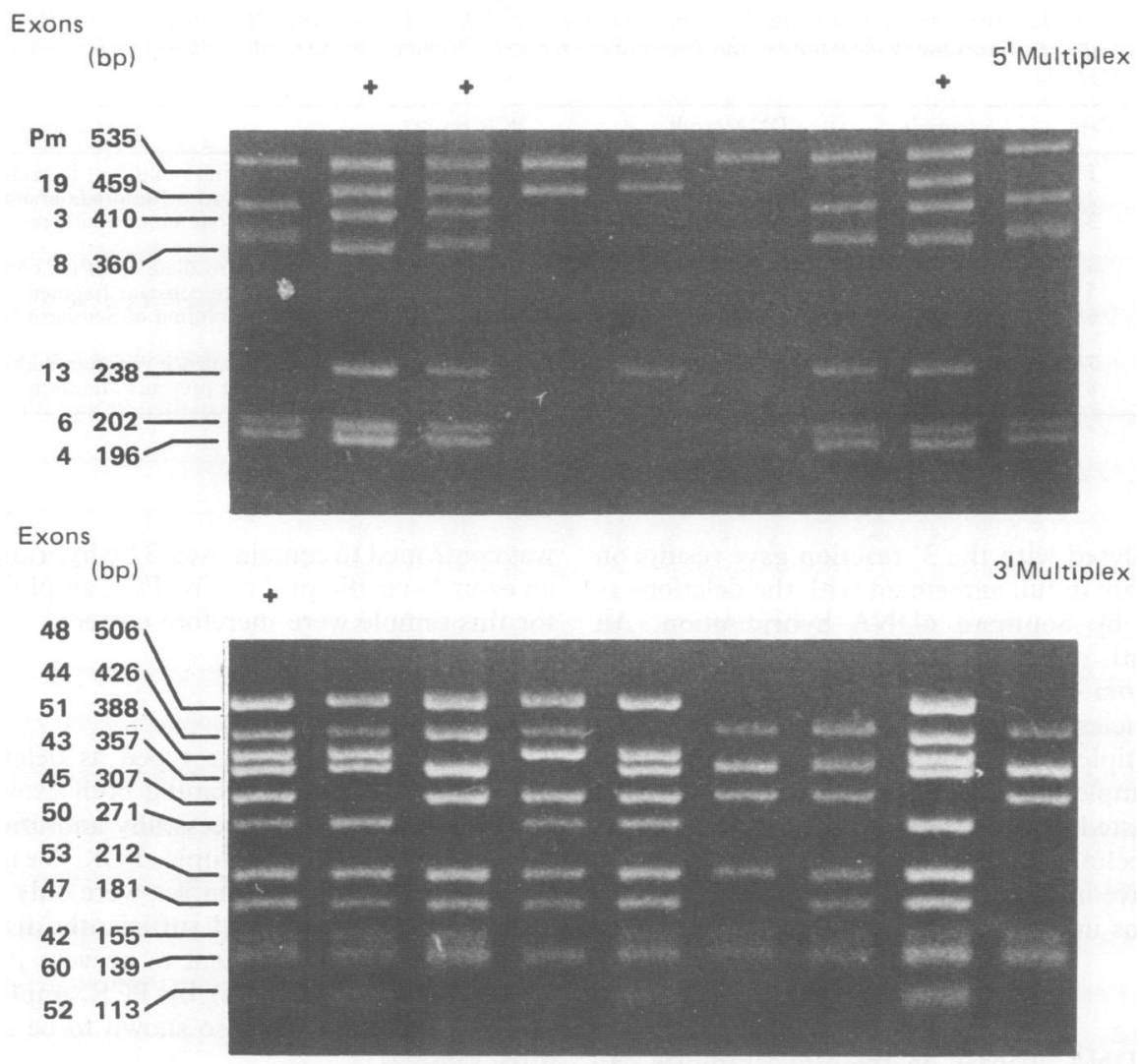

Figure 2 Products of amplification from the $5^{\prime}$ and $3^{\prime}$ multiplex reactions. Examples of normal controls $(+)$ and several deleted samples analysed by horizontal polyacrylamide gel electrophoresis, stained with ethidium bromide. The sizes of amplification products are shown in base pairs $(b p)$.

exon was amplified simultaneously with a control exon (remote from the exon under investigation) in a total reaction volume of $25 \mu$, using 1.5 units of $T a q$ DNA polymerase.

\section{SOUTHERN CDNA HYBRIDISATION}

The deletions had previously been characterised by Southern hybridisation of cDNA clones to genomic DNA. ${ }^{3}$ For more detailed analysis of samples showing discrepant results, exon specific sequences were produced by PCR amplification and used as probes. Exon 1 (223 bp product) and exons 8+9 (311 bp product) were amplified from cDNA clone 9-7 (cDMD 1-2a), ${ }^{1}$ with primers designed using the cDNA sequence, ${ }^{13}$ and an exon 3 specific sequence was amplified from genomic DNA with the primers used in the $5^{\prime}$ multiplex reaction. The products of amplification were purified using Quiagen PCR purification tips (Diagen), before labelling and hybridisation by standard methods.

\section{SEQUENCING}

Genomic DNA samples from case 8 and a control were amplified by PCR with primers $44 \mathrm{Fl}$ and $44 \mathrm{R}$. The sequence homologous to $44 \mathrm{Fl}$ is located 48 base pairs upstream from the location of primer $44 \mathrm{~F}$ in genomic DNA. ${ }^{8} 44 \mathrm{~F}$ and $44 \mathrm{R}$ are the primers incorporated in the $3^{\prime}$ multiplex reaction. The products of amplification were purified with Quiagen PCR purification tips (Diagen) and then sequenced directly ${ }^{14}{ }^{15}$ using Sequenase and an internal primer located in exon 44 .

\section{Results}

The $5^{\prime}$ and $3^{\prime}$ multiplex reactions amplify 7 and 11 exon specific sequences in a normal dystrophin gene, respectively (see control samples in fig 2). All amplifications give bands that are readily resolved by horizontal electrophoresis in polyacrylamide minigels.

In the comparative analysis of known mutations, 41 of 48 samples tested with the $5^{\prime}$ reaction and all 50 
Table 3 Details of the discrepant results found between Southern cDNA hybridisation and PCR amplification data. The samples are classified into groups according to the nature of the discrepancy (see text). Numbers in the results columns refer to exons. $\mathcal{F}=a b e r r a n t$ sized junction fragment.

\begin{tabular}{|c|c|c|c|c|c|}
\hline Group & Case & Sample & cDNA result & PCR result & Explanation \\
\hline 1 & $\begin{array}{l}1 \\
2 \\
3\end{array}$ & $\begin{array}{r}163 \\
2012 \\
470\end{array}$ & $\begin{array}{l}1-13 \text { deleted } \\
1-16 \text { deleted } \\
3-7 \text { deleted }\end{array}$ & $\begin{array}{l}1 \text { present, } 2-13 \text { deletion } \\
1 \text { present, 2-16 deletion } \\
3 \text { present, } 4-7 \text { deletion }\end{array}$ & $\begin{array}{l}\text { Failure to detect hybridisation } \\
\text { signal on autoradiograph. Low } \\
\text { resolution of Southern analysis }\end{array}$ \\
\hline 2 & $\begin{array}{l}4 \\
5 \\
6\end{array}$ & $\begin{array}{r}535 \\
2223 \\
1707\end{array}$ & $\begin{array}{l}8 / 9 \text { deleted } \\
8 / 9 \text { deleted } \\
8 / 9 \text { deleted }\end{array}$ & $\begin{array}{l}\text { No deletion } \\
\text { No deletion } \\
\text { No deletion }\end{array}$ & $\begin{array}{l}\text { Rare allele of RFLP comigrates } \\
\text { with constant fragment. Low } \\
\text { resolution of Southern analysis }\end{array}$ \\
\hline 3 & $\begin{array}{l}7 \\
8\end{array}$ & $\begin{array}{r}2268 \\
696\end{array}$ & $\begin{array}{l}6 / 7 \text { deleted }+\mathrm{J} \\
\text { No deletion }\end{array}$ & $\begin{array}{l}6 \text { present, } 3+7 \text { deleted } \\
44 \text { deleted }\end{array}$ & $\begin{array}{l}\text { Sequence variation within priming } \\
\text { site prevents annealing of primer }\end{array}$ \\
\hline
\end{tabular}

samples analysed with the $3^{\prime}$ reaction gave results on PCR that were in full agreement with the deletions as determined by Southern cDNA hybridisation. All exons in both multiplex reactions were successfully amplified from 49 of the 50 non-deleted samples.

Discrepancies between the Southern cDNA data and the multiplex PCR results were observed in a total of eight samples. These discrepancies are detailed below and listed in table 3. None of the errors was the result of technical inadequacy, observer error, or administrative mistakes; all were traceable to systematic problems inherent in the techniques being used.

\section{CASES 1 AND 2}

Southern cDNA analysis on these two samples had shown them to be deleted for exons 1 to 13 and 1 to 16, respectively. However, amplification of the muscle specific promoter and $5^{\prime}$ end of exon 1 in the $5^{\prime}$ multiplex PCR was successful in both cases, suggesting deletions of exons 2 to 13 and 2 to 16 , respectively. The two samples were investigated further by hybridisation of an exon 1 specific probe to several restriction enzyme digests (HindIII, BglII, $P s t \mathrm{I}, X m n \mathrm{I}$ ) and in all cases the probe hybridised to a fragment of the expected size, as observed in controls. The original data, obtained with a full XJcDNAl probe, ${ }^{16}$ had thus failed to detect a signal from the hybridisation to the intact exon 1.

\section{CASE 3}

A deletion of exons 3 to 7 on cDNA analysis showed as a deletion of exons 4 and 6 on PCR, but exon 3 amplified normally. The sample was tested with a different pair of primers, specific to the cDNA sequence for exon 3, and again the exon was successfully amplifed. Southern hybridisation was repeated using clone 9-7 (cDMD 1-2a) to probe new $E c o R I$ and $B g I I I$ digests and an aberrant sized fragment giving a weak hybridisation signal was observed that had not been detected previously. This was confirmed to contain exon 3 by hybridisation with an exon 3 specific probe. The PCR amplification data for this sample were therefore correct.

CASES 4, 5, AND 6

These three samples were typed as deleted for the single HindIII band containing both exons 8 and 9; however, exon 8 was successfully amplified from all three samples in the $5^{\prime}$ multiplex PCR. We investigated the possibility that the samples were only deleted for exon 9 (and that this had sufficiently disrupted the HindIII restriction fragment to prevent its detection on the Southern analysis) by PCR amplification of exon 9. This exon was also shown to be intact in all three samples.

No deletion was present in these three cases. The results were presumed to be because of the presence of a rare $8.3 \mathrm{~kb}$ allele of a HindIII polymorphism ${ }^{17}$ which comigrates with an $8 \mathrm{~kb} H$ indIII fragment detected by cDNA clone 9-7 (cDMD 1-2a), such that it is not readily resolved by conventional Southern analysis. This was confirmed by hybridising a Southern blot (HindIII digest) with a labelled sequence specific for exons 8 and 9. The resulting autoradiograph showed hybridisation to a larger HindIII restriction fragment of $8.3 \mathrm{~kb}$ in these three samples, compared with the normal $7.5 \mathrm{~kb}$ fragment in controls.

Three other samples, deleted only for the HindIII fragment that contains exons 8 and 9, and four samples in which the deletion started at this fragment and extended distally, were confirmed to be deleted for both exons 8 and 9 by PCR analysis.

\section{CASE 7}

On Southern cDNA analysis this sample was deleted for exons 6 and 7, with the presence of a junction fragment. On the 5 ' multiplex PCR analysis a deletion of exon 3 was detected, but not of exon 6. Further hybridisations with cDNA clone 9-7 (cDMD 1-2a) to several restriction enzyme digests $(E c o R I, B g l \mathrm{II}$, and 
Anneal temperature

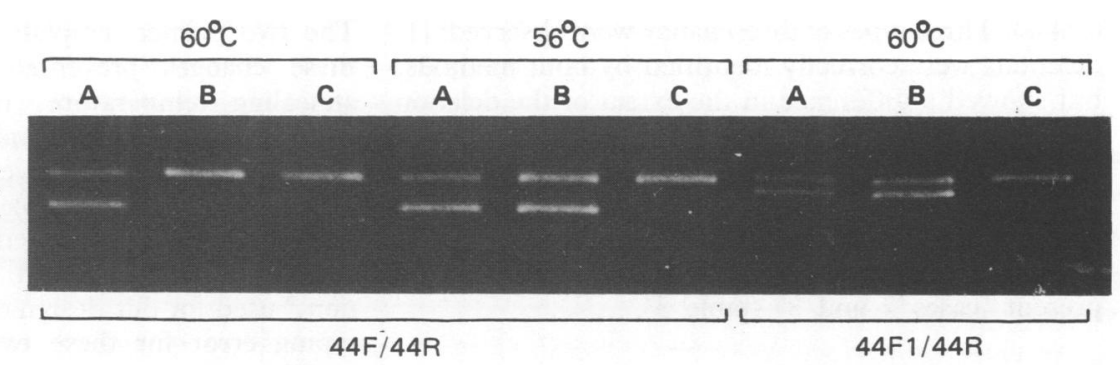

Exon 44 primers

$44 \mathrm{~F} / 44 R$

$44 \mathrm{~F} 1 / 44 \mathrm{R}$

Figure 3 Ethidium bromide stained minigel of PCR amplification products showing the effects of a sequence variation within the region homologous to a primer. At a high annealing temperature, exon 44 does not amplify in case 8. Reduction of the annealing temperature or use of a different forward primer in this example allows successful amplification. Exon 48 is used as a control amplification. Samples: $A=$ normal positive control; $B=$ case $8 ; C=$ control exon 44 deletion.

$X m n I$ ) confirmed the original result obtained with HindIII. However, using the full cDNA clone it was not possible to distinguish which of the two exons (6 or 7) was present in the junction fragment. This was investigated by probing the original HindIII filter with an exon 6 specific probe. The probe hybridised to the junction fragment in the affected boy and to both the normal sized fragment and junction fragment in his carrier mother. Additional PCR amplifications using different oligonucleotide primers specific for the cDNA sequences of exons 3 and 7 confirmed that exon 3 was present and exon 7 was deleted. A PCR amplification using the original primers that had failed to amplify exon 3 was then performed with a reduced annealing temperature of $56^{\circ} \mathrm{C}$ used in the cycling conditions. The amplification was successful with this reduction in annealing temperature.

\section{CASE 8}

This sample, which was known not to show a deletion by cDNA analysis, failed to amplify exon 44 in the $3^{\prime}$ multiplex reaction. Repeated hybridisation with cDNA clone 47-4(cDMD 56-7) on different restriction enzyme digests failed to detect a deletion. The PCR amplification of exon 44 was then repeated with a reduced annealing temperature as used in the previous case (case 7) and again this resulted in the successful amplification of the exon (fig 3).

The correlation between annealing temperature and success of amplification in both these samples suggested that unusual polymorphisms residing within the sequences complementary to the oligonucleotide primers were preventing successful annealing of those primers at the higher temperature, thereby preventing effective amplification. The problem in case 8 was localised to the forward primer (44F) by repeating the amplification of exon 44 with different combinations of primers at the original annealing temperature of $60^{\circ} \mathrm{C}$ (fig 3). By amplifying and directly sequencing across the region homologous to primer $44 \mathrm{~F}$, case 8 was found to differ from the primer sequence in two respects, with a G-A transition and $\mathrm{C}-\mathrm{G}$ inversion, as shown below.

Case 8: 5'GTTGTATGTACATGCTAGGTGTGTA

Primer

44F: 5'GTTGTGTGTACATCGTAGGTGTGTA

A normal control sample was also shown to have the C-G inversion. Replacement of the original primer $44 \mathrm{~F}$ with $44 \mathrm{~F} 2$, which incorporates the $\mathrm{C}-\mathrm{G}$ inversion as found in the sequencing reactions, facilitated the successful amplification of exon 44 from case 8 at $60^{\circ} \mathrm{C}$.

\section{Discussion}

An evaluation of 495 separate deletions (our deletion data [222] plus data from Koenig et al ${ }^{12}$ [273]) suggests that the $5^{\prime}$ and $3^{\prime}$ multiplex reactions will detect approximately $22 \%$ and $77 \%$ of deletions, respectively (table 1$)$. Six $(1 \cdot 2 \%)$ deletions extend across the regions covered by both reactions, giving a total detection frequency of $97 \cdot 7 \%$. The multiplex reactions of Chamberlain $e t a l^{8}$ and Beggs et al ${ }^{11}$ would together detect $94.5 \%$ of these deletions. The detection of an extra $3.2 \%$ by our reactions is the result of additional amplifications of exons $\mathbf{4 2}$ and 53.

Although the reactions of Chamberlain $e t a l^{8}$ and Beggs et al ${ }^{11}$ complement each other and amplify different exons, they overlap in the regions of the gene covered (fig 1). In most cases, mapping the end points of a deletion therefore requires both reactions to be performed on a sample. The modification to separate $5^{\prime}$ and $3^{\prime}$ reactions will reduce this need to perform a second amplification.

Our analysis of known deletion samples by multiplex PCR amplification showed agreement with the Southern cDNA data for all the $3^{\prime}$ deletions (50/50), but only $41 / 48$ of the $5^{\prime}$ deletions and $49 / 50$ of the non-deleted samples.

Discrepancies were found between the results obtained by the two methods of analysis in eight cases 
(5.4\%). Three types of discrepancy were observed: (1) deletions were correctly identified by both methods, but showed a difference in the extent of the deletion (cases 1 to 3); (2) the Southern cDNA analysis incorrectly identified deletions, which were correctly diagnosed as non-deletions by PCR amplification (cases 4 to 6); and (3) PCR analysis incorrectly detected deletions of sequences that were known to be present (cases 7 and 8) (table 3).

\section{EXTENT OF DELETIONS}

Southern hybridisation analyses, carried out with probe XJcDNAl in our laboratory (case $3^{18}$ and case 2 ) and a collaborating laboratory (case $1^{16}$ ), suggested that the deletions extended 5 ' to the first exon in two cases (1 and 2), or to exon 3 in another case (3), whereas these exons amplified successfully on PCR. The failure to detect the exons on the Southern analyses was presumably because of either poor transfer of the DNA in the blotting process, or the presence of undetected air bubbles during filter hybridisation. The presence of a deletion in these cases was correctly diagnosed, since several restriction fragments were deleted. However, if other fragments had not been deleted a diagnostic error could have occurred. Single fragment deletions clearly call for extra care in diagnosis.

The reclassification of the 3-7 deletion (case 3 ) as a 4-7 deletion does not explain the mild phenotype observed in this patient. Both a 3-7 and a 4-7 deletion would be expected to disrupt the translational reading frame of the dystrophin messenger RNA and result in the more severe Duchenne phenotype, ${ }^{18} 19$ but this patient has been classified as having a severe Becker phenotype. The two patients deleted for exons 2 to 13 and 2 to 16 respectively both have a Duchenne phenotype, as would be predicted from their deletions which disrupt the translational reading frame.

MISTAKEN DIAGNOSIS OF DELETION BY CDNA ANALYSIS Three samples (cases 4, 5, and 6) were incorrectly typed as deleted for exons $8 / 9$ by the method of Southern cDNA hybridisation. This resulted from a failure to resolve the rare allele of a polymorphism on the Southern blot, since it had comigrated with a constant fragment also detected by the cDNA probe. The observed frequency of this allele among our population of DMD/BMD patients is $4 / 283(1 \cdot 4 \%)$, substantially lower than the reported frequency of $10 \% .^{17}$

ERRORS IN DELETION DIAGNOSIS BY PCR AMPLIFICATION Two different exons failed to amplify from two samples that were shown to be intact at these loci (cases 7 and 8). In case 8, two sequence variations were found within the region complementary to primer $44 \mathrm{~F}$, a $\mathrm{C}-\mathrm{G}$ inversion and a $\mathrm{G}-\mathrm{A}$ transition.
The two primer template mismatches produced by these changes prevented priming at a stringent annealing temperature, resulting in a failure to amplify exon 44 and the misdiagnosis of a deletion in this sample. The C-G inversion was also found to be present in a control sample which we sequenced, and in the sequence deposited in the EMBL/GenBank database. This suggested that the original publication, ${ }^{8}$ used for our design of primer $44 \mathrm{~F}$, contained a typing error for these two bases. Using a primer (44F2) with a sequence that incorporated the $\mathrm{C}-\mathrm{G}$ inversion (but still produced a mismatch at the site of the G-A transition), exon 44 was successfully amplified from case 8 at the standard annealing temperature. We have subsequently replaced $44 \mathrm{~F}$ with $44 \mathrm{~F} 2$ in the $3^{\prime}$ multiplex reaction.

Two separate observations were made in case 7. Firstly, the successful amplification of exon 3 from this sample was shown to be dependent on the use of a reduced annealing temperature, again suggesting that a sequence variation was preventing one of the primers from annealing to the template. Secondly, the enhanced resolution of the PCR technique enabled us to show that exon 7 was deleted and that exon 6 was present in the junction fragment seen on the Southern analysis.

The possibility of a sequence variant residing within a priming site is an inherent problem associated with the PCR technique. This potentially hazardous problem, which we have termed 'NAFNAP' (Non$A$ mplification From Non-Annealing of a Primer), has been reported for the $D 7 S 8$ locus that is closely linked to cystic fibrosis ${ }^{20}$ and we have also detected the problem with the amplification of the pERT87-15/ XmnI (DXS142) polymorphic locus. ${ }^{21}$ This locus failed to amplify from seven out of 100 male samples known to be intact for DXS142, and from three out of 25 unrelated, known heterozygous females typed as homozygous when analysed by PCR. ${ }^{22}$

We have been able to overcome the problem by using a reduced annealing temperature, which would be expected to reduce the sequence specificity required for primer hybridisation. However, adjustment of annealing temperature will not necessarily correct for all types of sequence variation that are found in the genome and for many reactions this is not a practical solution since amplification of unwanted, non-specific sequences is likely to occur.

The false identification of an apparent single exon deletion (groups 2 and 3 ) is diagnostically much more hazardous than errors in defining the extent of deletions (group 1). Where two or more restriction fragments fail to hybridise to a probe it is reasonable to assume the presence of a deletion. Similarly, the probability of finding two primer failures in one subject would be so low that where two or more contiguous sequences fail to amplify a true deletion can reasonably be assumed. 
In view of the $8 / 148(5.4 \%)$ mistypings observed in our study, we recommend the following procedures in order to reduce errors that may lead to misdiagnosis. (1) Since the two methods of analysis are vulnerable to different types of error, any future diagnosis in a family should be performed either using the technique which was originally used to define the mutation, or the data should first be verified using the new technique. (2) To circumvent possible inaccuracies in defining the extent of a deletion, owing to the presence of junction fragments that may hybridise weakly or not be readily resolved by Southern hybridisation, diagnostic tests based on a deletion should be performed on central regions within the deletion, rather than at the extremities of the mutation. (3) To avoid misdiagnosis of a deletion in subjects exhibiting non-hybridisation or nonamplification of a single sequence, the deletion should be verified either by performing further hybridisations on different restriction enzyme digests, or by PCR amplification using a different pair of primers specific for that same region. From the analysis of 495 separate deletions (table 1) this would involve approximately $28 \%$ of PCR detectable deletions. (4) Every set of PCR primers introduced into diagnostic work should be stringently monitored by testing a large number of subjects for sequence variants which interfere with priming.

A disadvantage of only using the method of PCR amplification for deletion detection is that duplications and diagnostically useful aberrant sized junction fragments will not be detected. However, the heterogeneity in the size and location of dystrophin gene deletions requires the use of at least six separate cDNA clones for their detection, ${ }^{1}$ in both a costly and time consuming procedure, compared with the technique of multiplex PCR amplification which allows the rapid detection of $98 \%$ of these deletions.

PCR amplification is rapid, cost effective, nonradioactive, and gives greater resolution than the Southern hybridisation technique. We conclude that provided suitable precautions are taken to circumvent possible mistypings, multiplex PCR amplification is the method of choice for the detection and mapping of dystrophin gene deletions.

We thank the Muscular Dystrophy Group of Great Britain and Northern Ireland and the Generation Trust for financially supporting this work; Professor V Dubowitz, Dr C Berry, and Dr S Hodgson for the referral of patients; Alison Coffey and Roland Roberts for providing some of the primers used in this study; and Charlotte Cole and Dr Peter Green for help and advice with sequencing.
1 Koenig M, Hoffman EP, Bertelson CJ, Monaco AP, Feener C, Kunkel LM. Complete cloning of the Duchenne muscular dystrophy (DMD) cDNA and preliminary genomic organisation of the DMD gene in normal and affected individuals. Cell 1987;50:509-17.

2 Forrest SM, Cross GS, Speer A, Gardner-Medwin D, Burn J, Davies KE. Preferential deletion of exons in Duchenne and Becker muscular dvstrophies. Nature 1987;329:638-40.

3 Hodgson S, Hart K, Abbs S, et al. Correlation of clinical and deletion data in Duchenne and Becker muscular dystrophy. f Med Genet 1989;26:682-93.

4 Hu X, Ray PN, Murphy E, Thompson MW, Worton RG. Duplicational mutation at the Duchenne muscular dystrophy locus: its frequency, distribution, origin and phenotype/ genotype correlation. Am f Hum Genet 1989;46:682-95.

5 Bakker E, Hofker MH, Goor N, et al. Prenatal diagnosis and carrier-detection of Duchenne muscular dystrophy with closely linked RFLP's. Lancet 1986;i:655-8.

6 Hodgson SV, Bobrow M. Carrier detection and prenatal diagnosis in Duchenne and Becker muscular dystrophy. Br Med Bull 1989;45:719-44.

7 Abbs S, Roberts RG, Mathew CG, Bentley DR, Bobrow M. Accurate assessment of intragenic recombination frequency within the Duchenne muscular dystrophy gene. Genomics 1990;7:602-6.

8 Chamberlain JS, Gibbs RA, Ranier JE, Nguyen PN, Caskey CT. Deletion screening of the Duchenne muscular dystrophy locus via multiplex DNA amplification. Nucleic Acids Res 1988;16: 11141-56.

9 Saiki RK, Scharf S, Faloona F, et al. Enzymatic amplification of alpha-globin genomic sequences and restriction site analysis for diagnosis of sickle cell anaemia. Science 1985;230:1350-4.

10 Chamberlain JS, Gibbs RA, Ranier JE, Caskey CT. Multiplex PCR for the diagnosis of Duchenne muscular dystrophy. In: Innis M, Gelfand D, Sninski J, White T, eds. PCR protocols: a guide to methods and applications. Orlando: Academic Press, 1989:272-81.

11 Beggs AH, Koenig M, Boyce FM, Kunkel LM. Detection of 98\% of $\mathrm{DMD} / \mathrm{BMD}$ gene deletions by polymerase chain reaction. Hum Genet 1990;86:45-8.

12 Koenig M, Beggs AH, Moyer M, et al. The molecular basis for Duchenne versus Becker muscular dystrophy: correlation of severity with type of deletion. Am J Hum Genet 1989;45: 498-506.

13 Koenig M, Monaco AP, Kunkel LM. The complete sequence of dystrophin predicts a rod-shaped cytoskeletal protein. Cell 1988;53:219-28.

14 Green PM, Bentley DR, Mibashan RS, Nilsson IM, Giannelli F. The molecular pathology of haemophilia B. EMBO F 1989;8: 1067-72.

15 Winship PR. An improved method for directly sequencing PCR amplified material using DMSO. Nucleic Acids Res 1989;17: 1266.

16 Burghes AHM, Logan C, Hu X, Belfall B, Worton RG, Ray PN. A cDNA clone from the Duchenne/Becker muscular dystrophy gene. Nature 1987;328:434-7.

17 Prior TW, Friedman KJ, Silverman LM. RFLP for HindIII at the Duchenne muscular dystrophy gene. Nucleic Acids Res 1989;17:2370.

18 Malhotra SB, Hart KA, Klamut HJ, et al. Frame-shift deletions in patients with Duchenne and Becker muscular dystrophy. Science 1988;242:755-9.

19 Monaco AP, Bertelson CJ, Liechti-Gallati S, Moser H, Kunkel LM. An explanation for the phenotypic differences between patients bearing partial deletions of the DMD locus. Genomics 1988;2:90-5.

20 Fujimura FK, Northrup H, Beaudet A, O'Brien WE. Genotyping errors with the polymerase chain reaction. $N$ Engl f Med 1990;322:61.

21 Roberts RG, Cole CG, Hart KA, Bobrow M, Bentley DR. Rapid carrier and prenatal diagnosis of Duchenne and Becker muscular dystrophy. Nucleic Acids Res 1989;17:811-2.

22 Clark S, Yau SC, Abbs S, et al. Experience of the polymerase chain reaction in the diagnosis of Duchenne and Becker muscular dystrophy: benefits and problems encountered. 7 Med Genet 1990;27:656A. 